\title{
NEW TAXA AND COMBINATIONS IN RUTACEAE
}

\author{
R. C. KAASTRA
}

Instituut voor Systematische Plantkunde, Utrecht

New taxa and combinations are published here in anticipation of the revision of the Rutaceae-Pilocarpinae to be published in the near future (thesis, and in Flora Neotropica). Two new combinations of species excluded from subtribe Pilocarpinae are added.

Esenbeckia pentaphylla (Macfadyen) Grisebach subsp. belizensis (Lundell) Kaastra, stat. nov.

Basionym: Esenbeckia belizensis Lundell, Contr. Univ. Mich. Herb. 6: 33. 1941.

Esenbeckia pentaphylla (Macfadyen) Grisebach subsp. australensis Kaastra, subsp. nov.

Arbor 10-15 m trunco 5-25 (-40?) cm diametro. Folia trifoliolata foliolis apice acuminato acumine ipso obtuso vel interdum aliquot apice obtuso vel rotundo. Inflorescentiae paniculatae laxiflorae. Flores circa $8 \mathrm{~mm}$ diametro, fragrantes. Petala 3.7-4.0 $\times 2.0-2.2 \mathrm{~mm}$, papyracea hemidiaphana, alba. Filamenta 3.5-4.0 mm longa. Discus circa $2.0 \mathrm{~mm}$ diametro, velut ovarium dense strigillosum pilis $0.1-0.3 \mathrm{~mm}$ longis. Fructus clausi $2.4-2.8 \times$ circa $2.9 \mathrm{~cm}$, fructus dehisci $3.4-4.0 \mathrm{~cm}$, ubi immaturi dense pilosi deinde glabrescentes. Semen nitidum area chalazina manifesta.

Typus: A. Dugand G. 536, Colombia, Atlántico, El Pajar forest, alt. 60 $300 \mathrm{~m}$, fr., 29 Mar. 1934 (HOLOTYPUS, F 726782; isotypi, G, US 2×).

The fully grown but not yet mature fruits of this subsp. are minutely pubescent, while those of the other subsp. are glabrous. For the rest, the fruits are much like those of subsp. pentaphylla, with its secondary apophyses.

Esenbeckia berlandieri Baillon ex Hemsley subsp. litoralis (Donnell Smith) Kaastra, stat. nov.

Basionym: Esenbeckia litoralis J. Donnell Smith, Bot. Gaz. (Crawfordsville) 23: 242. 22 Apr. 1897.

Esenbeckia berlandieri Baillon ex Hemsley subsp. acapulcensis (Rose) Kaastra, stat. nov.

Basionym: Esenbeckia acapulcensis Rose, Contr. U. S. Natl. Herb. 5: 111. t. 3. f. 1-7. 1897.

Esenbeckia feddemae Kaastra, spec. nov., fig. 1 .

Frutex vel arbor parva. Folia alterna (tri-, quadri-) quinquefoliolata. Petiolus semiteres canaliculatus, $3-8 \mathrm{~cm}$ longus, pilosus. Petiolulus usque ad 


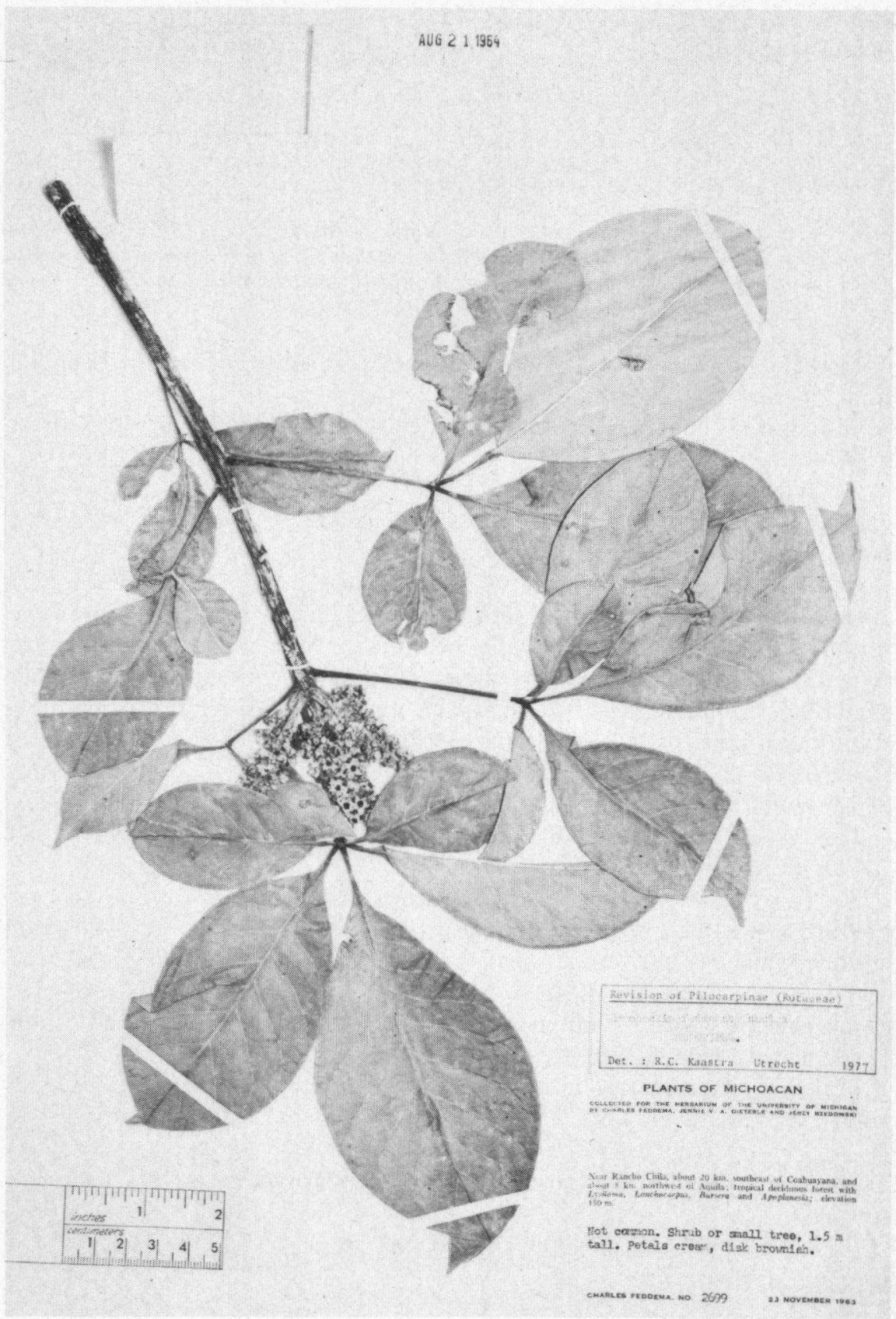

Fig. 1. Esenbeckia feddemae Kaastra, holotype (MICH). 
$1 \mathrm{~cm}$ longus. Lamina foliolorum elliptica vel parum obovata basi (longe) attenuata gradatim decurrenti in petiolulum, foliolorum lateralium basi inaequali, apice obtuso rotundo vel parum acuminato saepe leviter recurvo, ad 7.5$18.5 \times 4.3-7.7 \mathrm{~cm}$, utrinque pilosa, praesertim subtus ad tactum mollis, vena media supra plana. Inflorescentiae terminales foliis brevissimae, pilosae. Flores 9.0-9.5 mm diametro, glabri. Lobi calycis aestivatione quincunciali, depresse ovati apice rotundo, coriacei margine pellucido-papyracea. Petala aestivatione quincunciali, ovata apice rotundo, pellucido-papyracea, cremea. Discus rosulaformis leviter decemlobatus, infuscatus. Carpella purpurea basi solum connata, postea apophyse glandibus tuberculisque glandulosis instructa. Fructus depressi stellatim quinquelobati truncati, $2.0-2.5 \mathrm{~cm}$ alti, glabri. Loculi cornu dorso-apicali erecto $1.0-1.3 \mathrm{~cm}$ longo muniti, carinati, obsolete muricati aculeis 1-2 mm longis, commissuris nervatis extus. Semina ignota.

Typus: C. Feddema 2699, Mexico, Michoacan, Rancho Chila, circa $20 \mathrm{~km}$ SE of Coahuayana, and circa $5 \mathrm{~km} \mathrm{NW}$ of Aquila, fl., 23 Nov. 1963 (HOLOTYPUs, $\mathrm{MICH}$; isotypus, $\mathrm{MICH}$ ).

This new species is close to E. berlandieri. The hairs in the vegetative parts are, however, longer and they are spreading. The inflorescences are shorter with larger flowers, while the carpels are provided with purple apophyses.

Esenbeckia collina Brandegee subsp. conspecta Kaastra, subsp. nov.

Frutex tenuis $3 \mathrm{~m}$ altus ramulis teretibus. Folia alterna vel subopposita petiolis porcatis foliolis lateralibus terminale minoribus, puberula pilis $0.3 \mathrm{~mm}$ longis supra plus minusve patentibus leviter curvatis infra adpressis. Paniculae frondosae terminales e partibus pluribus paniculatis erectis vel patentibus compositae, quae $6-7 \times 4 \mathrm{~cm}$ et basi foliiferae, pedicellis $3.0-3.5 \mathrm{~mm}$ longis. Flores 10.5-11.0 mm diametro, odore carentes. Lobi calycis latissime vel depresse ovati. Petala 4.8-5.0 $\times 2.5 \mathrm{~mm}$. Antherae $2.0 \times 1.3-1.5 \mathrm{~mm}$ circumferentia, cum apice mucronato $0.1 \mathrm{~mm}$ longo. Apophyses carpellorum 1.2-1.3 $\times 0.8$ $\mathrm{mm}$ magnae iam in alabastro magnitudinis illius, tuberculis paucis $0.1-0.2 \mathrm{~mm}$ longis obsitae. Stylus $3.2 \mathrm{~mm}$ longus, parte libera $2.3 \mathrm{~mm}$. Fructus ignoti.

TyPUS: B. L. Turner 2086, Mexico, Michoacan, 2 miles NW of La Placita, 45 air miles S of Colima, fl., 5 Jul. 1950 (HOLOTYPUS, MICH).

Compared with subsp. collina, this new subsp. has a less compact growth with its non crowded leaves. The venation of the leaflets is more strongly prominent, the flowers are larger and they seem to be darker, while the apophyses are smoother. There is some resemblance to E. warscewiczii in the filaments, the glabrous perianth, the longer flower organs, etc.

\section{Esenbeckia irwiniana Kaastra, spec. nov., fig. 2.}

Frutex pauciramosus vel arbor parva. Folia alterna trifoliolata foliolis petiolulatis. Petiolus semiteres canaliculatus, $2-6 \mathrm{~cm}$ longus, dense pilosus. Petiolulus 5-11 mm, foliolorum lateralium 1-5 mm. Lamina foliolorum obovata vel elliptica basi rotunda obtusa vel brevissime attenuata, foliolorum lateralium inaequali, apice emarginato vel obtuso, $4-11 \times 2.2-5.0 \mathrm{~cm}$ vel minor, 


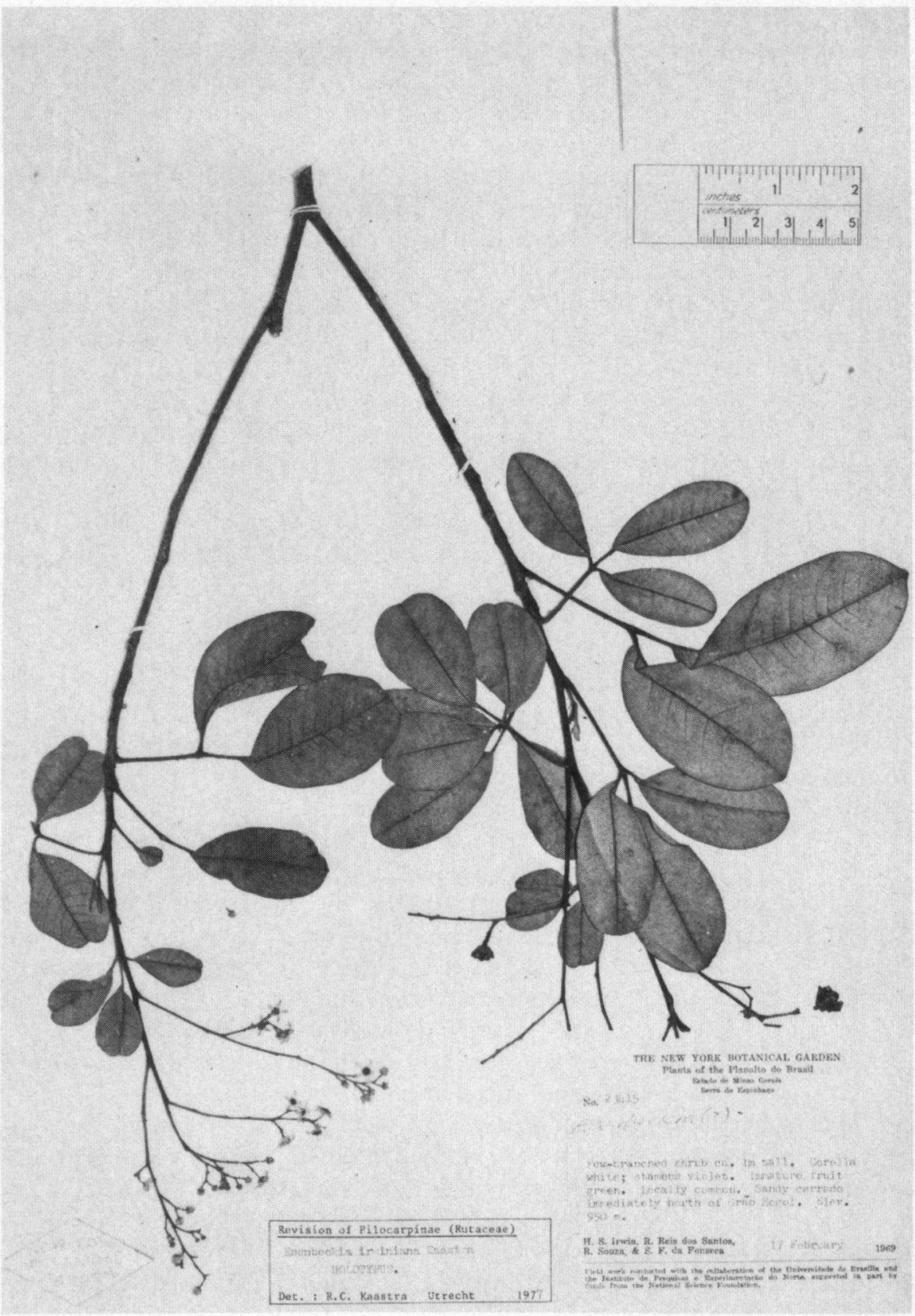

Fig. 2. Esenbeckia irwiniana Kaastra, holotype (NY). 
chartacea, supra pilosa infra pilosa vel tomentosa pilis curvatis mollibus, vena media impressa in paginam superiorem. Inflorescentiae terminales paniculatae e pedunculis usque ad duos compositae, foliis longiores, dense pilosae. Flores 8.5-9.0 mm diametro. Lobi calycis aestivatione quincunciali, late ovati ad subcirculares, obtusi, crassissime coriacei, infra pilosi. Petala aestivatione cochleari vel quincunciali, elliptica vel subobovata, obtusa vel rotunda, plus minusve pellucido-papyracea, sulphurea, supra papillosa infra pilosa. Discus cupulatus leviter lobatus, carnosus, glaber. Carpella connata, glabra, demum tuberculis obsita et incana. Fructus depresse globosi stellatim quinquelobati, $1.5-2.0 \mathrm{~cm}$ alti, incani. Loculi apophyse dorso-apicali $4-6 \mathrm{~mm}$ longa instructi, irregulariter muricati. Semen lacrimiforme basi-ventraliter complanatum apice breve curvirostrato. Embryo unus, punctatus, cotyledonibus inaequalibus.

TyPus: H. S. Irwin, R. Reis dos Santos, et al. 23415, Brazil, Minas Gerais, Serra do Espinhaço, N of Grão Mogol, fl., 17 Feb. 1969 (HOLOTYPUs, NY; isotypus, $\mathrm{U}$ ex NY).

This species is close to E. pumila, but it can be distinguished vegetatively by the slender petioles and the stalked leaflets.

Esenbeckia grandiflora Martius subsp. grandiflora var. intermedia (Martius ex Engler in Martius) Kaastra, stat. nov.

Basionym: Esenbeckia intermedia Martius ex Engler in Martius, Fl. bras. 12(2): 147. t. 32. f. 2. 1874.

Esenbeckia grandiflora Martius subsp. brevipetiolata Kaastra, subsp. nov.

Frutex 1-3 m. Petiolus 2-5 (-8) mm longus. Lamina folioli 4.0-9.5 $\times 2.2$ $5.0 \mathrm{~cm}$ vel minor, venis utrinque prominentibus. Inflorescentiae $3-5 \times 2 \mathrm{~cm}$. Bracteae circa $0.8 \times 0.1 \mathrm{~mm}$. Flores $7.5-8.0 \mathrm{~mm}$ diametro, virides, omnino glabri. Lobi calycis $0.5-0.6 \times 1.0-1.4 \mathrm{~mm}$. Petala $3.5 \times 2.0 \mathrm{~mm}$, coriacea. Antherae non cucullatae, $0.7 \times 0.6 \mathrm{~mm}$, non tumidissimae. Discus sulcatus usque ad $1 / 6$ crassitudinis. Carpella circa $0.7 \mathrm{~mm}$ alta, tuberculis oblongis $0.2-0.3 \mathrm{~mm}$ longis obsita. Fructus aculeis paulo brevibus angulato-pyramidalibus circa 2.5 $\mathrm{mm}$ longis armati, minus echinati quam in subspecie grandiflora.

Typus: R. P. Belem \& M. Magelhães 914, Brazil, Bahia, Maraú, fl., 25 Apr. 1965 (HOLOTYPUS, NY ex UB).

Subspecies brevipetiolata resembles collections of E. grandiflora var. grandiflora from the eastern Brazilian coasts in a similar texture of the leaves. This may be due to the same ecological conditions. It differs by the shorter petioles and the smaller, glabrous flowers.

\section{Esenbeckia oligantha Kaastra, spec. nov., fig. 3.}

Frutex vel arbor parva, adpresso-pubescens pilis cremeis in partibus vegetativis praeter ramulos vetos. Folia alterna, simplicia. Petiolus in pagina adaxiali leviter planatus, $2-15 \mathrm{~cm}$ longus. Lamina elliptica vel obovata, basi rotunda vel obtusa inaequali, apice obtuso vel subacuminato, 4-11 $\times 2.5-8.5$ $\mathrm{cm}$, subcoriacea, costa supra plana deinde costata. Paniculae frondosae termi- 


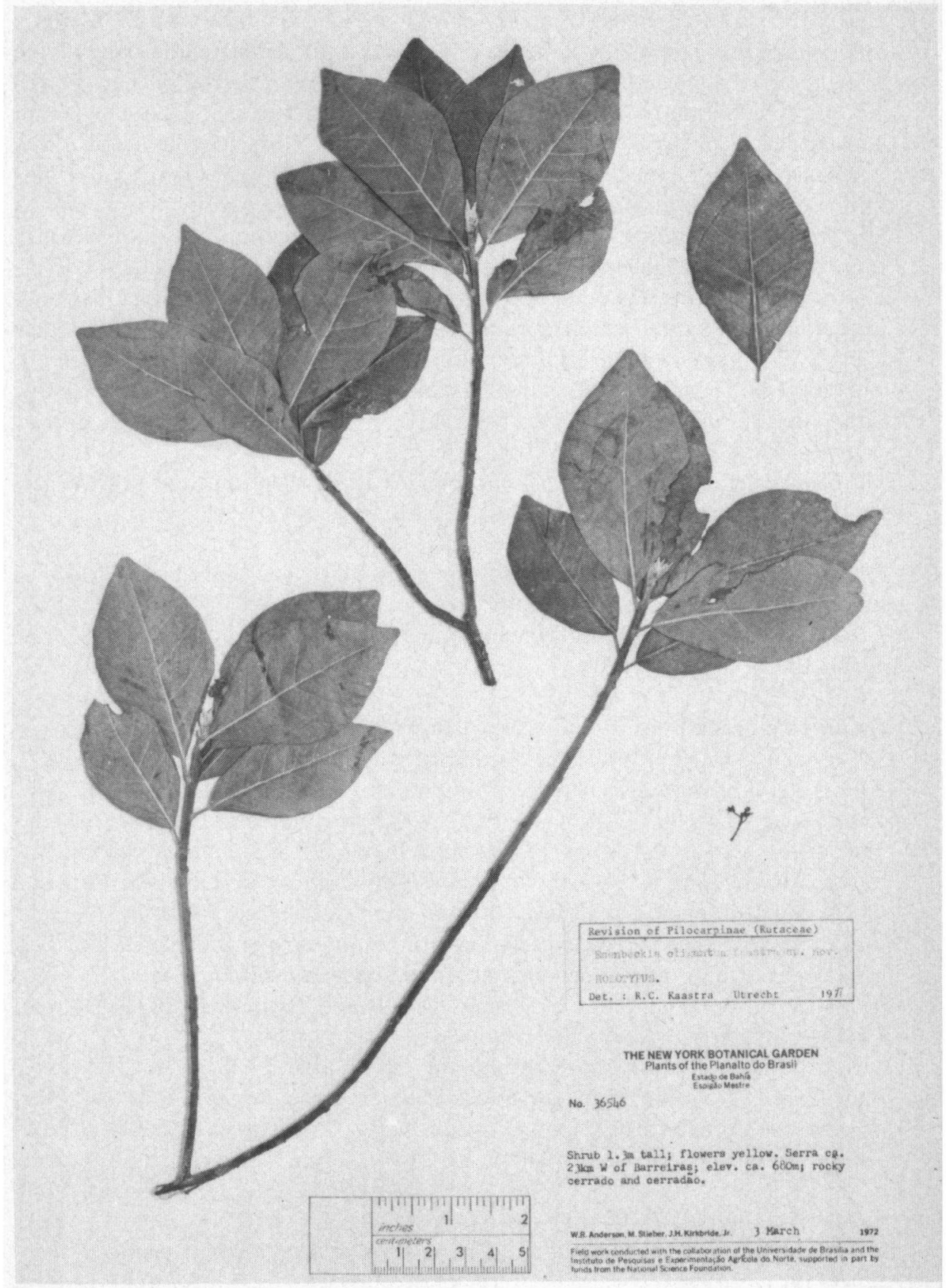

Fig. 3. Esenbeckia oligantha Kaastra, holotype (U). 
nales floribus paucis, foliis breviores, indumento illi partium vegetativarum simili. Flores 5-7-meri, circa 5.0-6.5 mm diametro. Lobi calycis aestivatione aperta, late triangulares, adpresse pubescentes infra et basi supra. Petala valvata, ovato-elliptica apice acuminato et uncinato, coriacea, incrassata versus apicem, flava, supra papillosa infra sparsim strigillosa. Discus annularis 5-7lobatus, glaber, laevis. Carpella connata, subglabra, apophyse apicali tuberculata instructa. Fructus 4-6-loculares, depressi stellatim lobati, $1.4-1.9 \mathrm{~cm}$ alti, incani. Loculi apophyse dorsali obtusa ad $3 \mathrm{~mm}$ longa ad circa $1 / 3$ ab apice instructi, obsolete tuberculati. Semen unum in quoque loculo. Embryones duo semine.

Typus: W. R. Anderson, M. Stieber, et al. 36546, Brazil, Bahia, Espigão Mestre, circa $23 \mathrm{~km} \mathrm{~W}$ of Barreiras, fl., 3 Mar. 1972 (HOLOTYPUS, U ex NY; isotypi, NY $9 \times$ ).

The present new species has much in common with E. cornuta and E. leiocarpa. There are, however, striking differences: the indument is creamish with the hairs less shining, the leaves are broader with a rounded or obtuse base, the inflorescences are quite different, as well as the aestivation. The fruits differ strongly from those of E. leiocarpa, but they are probably more similar to the poorly known fruits of $E$. cornuta.

Esenbeckia pilocarpoides Kunth subsp. maurioides (Martius) Kaastra, stat.nov.

Basionym: Esenbeckia maurioides Martius, Nov. gen. sp. 3: 82.t. 232. 1831.

Synonym: Esenbeckia pilocarpoides Kunth var. maurioides (Martius) Engler in Martius, Fl. bras. 12(2): 144. 1874.

Esenbeckia amazonica Kaastra, spec. nov., fig. 4.

Arbor parva vel frutex. Folia alterna, aliquando aliquot folia subopposita, unifoliolata foliolo sessili. Petiolus plus minusve teres, alatus, $11-50 \mathrm{~mm}$ longus, glaber. Lamina folioli obovata basi vulgo cuneata vel paulum brevissime attenuata, apice (sub)acuminato acumine 6-9 (-16) mm longo, 8-31 $\times 2.8$ $12.5 \mathrm{~cm}$, chartacea, utrinque subglabra. Inflorescentiae terminales, circa foliorum longitudine, minute pubescentes. Flores (7-) 8-14 mm diametro. Lobi calycis aestivatione quincunciali, latissime ovati vel cordiformes, obtusi, per anthesin ex purpureo virides, utrinque glabri vel infra minute pubescentes. Petala aestivatione imbricata, ovata, obtusa, chartacea, viridi-alba basi et margine supra purpurea vel atrorubra, utrinque minute pubescentia vel infra glabra. Filamenta appendice basali radiali plus minusve globulosa instructa, glabra. Discus annularis quinquepartitus, carnosus, purpureus, granulatus et parum tuberculatus, glaber. Carpella vix connata, in parte supera apophyse parva instructa, tuberculis obsita, (sub)glabra. Fructus depressi stellatim quinquelobati, $1.5-2.5 \mathrm{~cm}$ alti, (sub)glabri. Loculi tantum connati in parte inferna, apophyse dorsali instructi ad $1 / 2-1 / 3$ ab basi, leviter tuberculati, extus prominenter nervati. Semen oblique lacriforme. Embryo unus, punctatus, cotyledonibus inaequalibus.

TYPUS: A. Ducke 1266, Brazil, Amazonas, Esperança, near mouth of Rio Ja- 


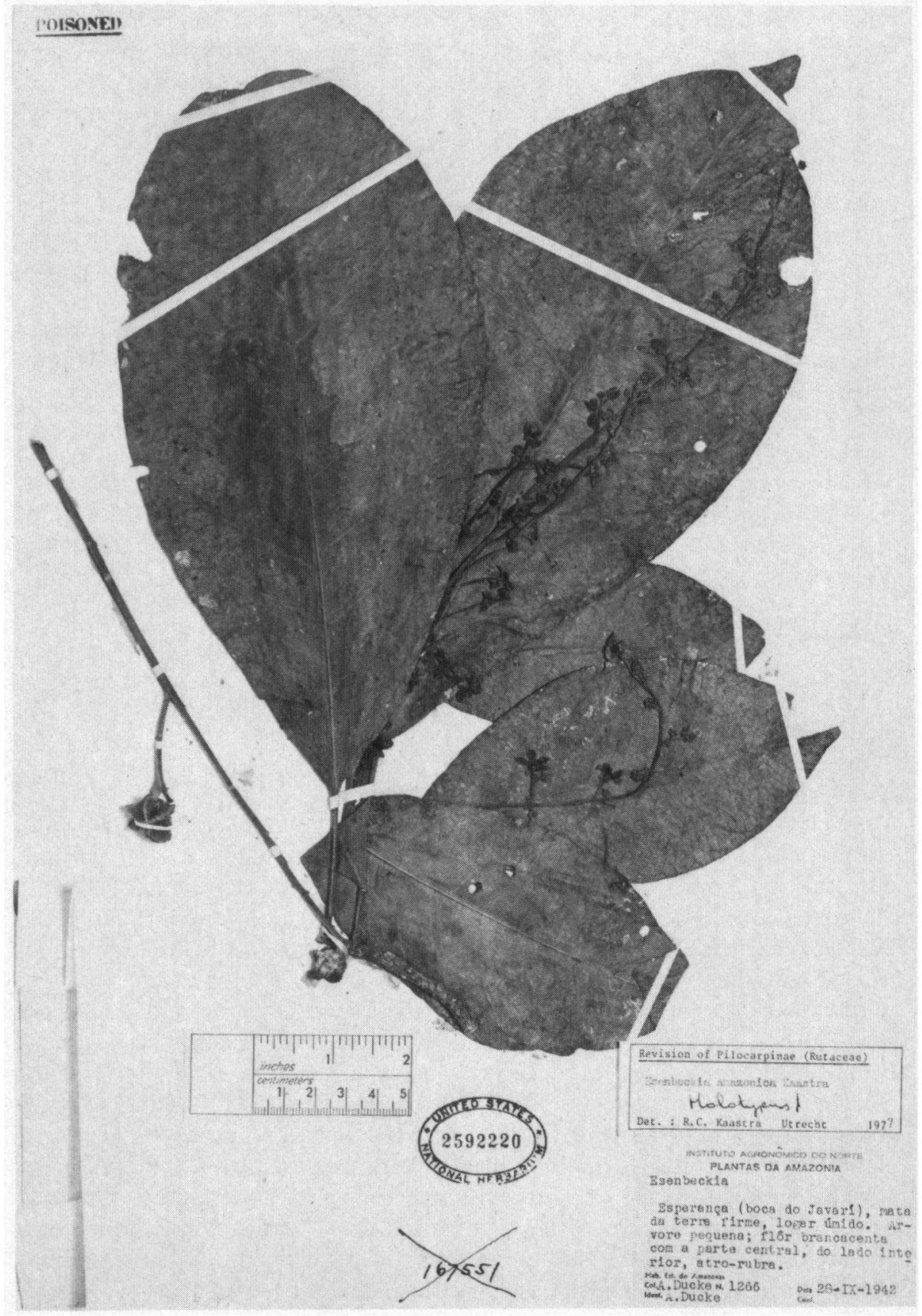

Fig. 4. Esenbeckia amazonica Kaastra, holotype (US). 
vary, fl. \& fr., 28 Sep. 1942 (HoLotypus, US 2592220; isotypi, A, F, NY, R $3 \times$, UC).

This species is close to E. pilocarpoides. It differs in the broader leaflets, in the inflorescences, which are twice as long and wide, in the flowers, which are larger in all aspects and different in colour, and in the smoother fruits.

Esenbeckia scrotiformis Kaastra, spec. nov., fig. 5.

Frutex vel arbor (?) indumento e pilis cremeis constanti. Folia alterna, trifoliolata. Petiolus semiteres canaliculatus, $4-9 \mathrm{~cm}$ longus. Petiolulus $0.5-10.0$ $\mathrm{mm}$ longus. Lamina foliolorum obovata vel elliptica basi attenuata in petiolulum decurrenti, foliolorum lateralium basi inaequalissima, apice breve acuminato acumine ipso emarginato vel retuso, $6.5-19.0 \times 2.5-9.0 \mathrm{~cm}$, chartacea, utrinque minute puberula, vena media supra plana, venis lateralibus infra prominentibus. Inflorescentiae terminales paniculatae ex inflorescentiis partialibus usque ad tres compositae, foliorum longitudine breviores, floribus paucis. Flores circa $9.5-12.0 \mathrm{~mm}$ diametro, proterandri. Lobi calycis aestivatione quincunciali, cordiformes, praeter basim versus membranacei, infra subglabri. Petala aestivatione valvata marginibus interioribus induplicatis, ovata apice ultimo acuminato, coriacea sed basi ad centrum teniora, supra atropurpurea dense villosa praeter basin ad centrum pilis unicellularibus lilacinis usque ad $1 \mathrm{~mm}$ longis, infra viridia glabra. Filamenta glabra, tumore basali radiali scrotiformi solido instructa. Discus cupulatus profunde incurvatus in quinque partes bilobatas, papillatus. Carpella immersa in discum, connata, glabra, apophyse apicali instructa. Fructus ignoti.

Typus: E. Ule 9501, Brazil, Acre, Seringal S. Francisco (near Rio Acre, above Iñapari), fl., Sep. 1911 (HOLOTYPUS, G; isotypi, K, L).

This species is distinct from all others in Esenbeckia by its villous corolla with valvate aestivation. It takes its name from the striking resemblance of the filaments to the genital parts of the human male.

Esenbeckia Kunth subgenus Oppositifolia Kaastra, subg. nov.

Folia (sub)opposita. Inflorescentiae terminales ramulis lateralibus (sub)oppositis. Flores proterandri. Lobi calycis pseudocosta glandulosa instructi. Petala aestivatione imbricato-valvata, nervatura camptodroma vel quodam modo actinodroma suprabasali vel parallela. Antherae in spatio $1 / 3-2 / 5(-1 / 2)$ a basi affixae. Semina plerumque duo in quoque loculo, area chalazina fusca, endospermii granularis reliquiis quantum scio.

SUBGENERITYPUS: Esenbeckia febrifuga (Saint-Hilaire) Jussieu ex Martius.

Apart from the type species the following species belong to this subgenus: $E$. densiflora (Chodat \& Hassler) Hassler, and E. hieronymi Engler.

Esenbeckia Kunth subgenus Lateriflorens Kaastra, subg. nov.

Folia alterna. Inflorescentiae laterales deminutae. Petala aestivatione valvata, unicostata. Antherae medio dorsifixae. Semen unum in quoque loculo observatum, area chalazina atrofusca, endospermio granulari destitutum. 


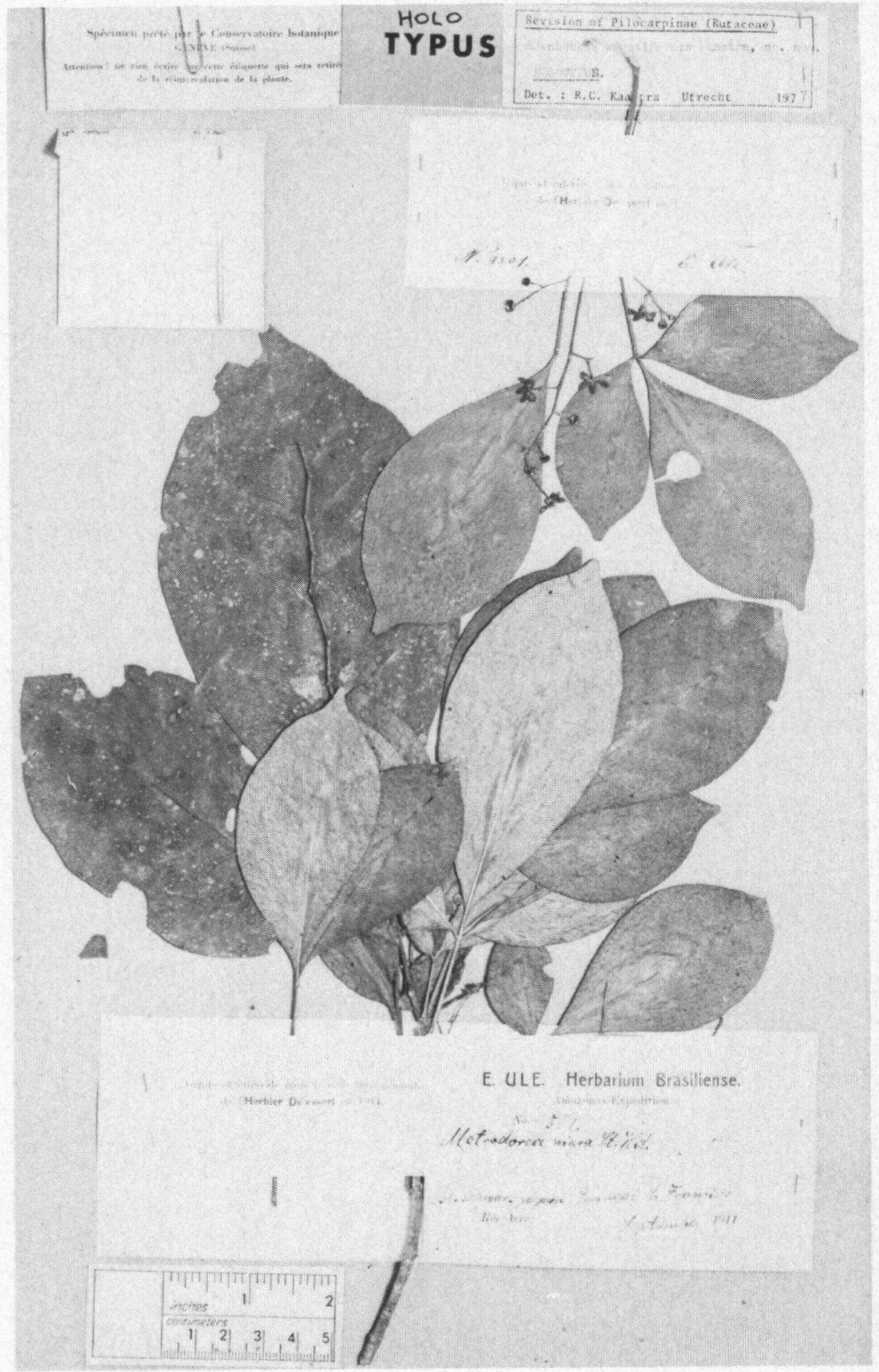

Fig. 5. Esenbeckia scrotiformis Kaastra, holotype (G). 
SUBGENERITYPUS: Esenbeckia almawillia Kaastra.

Esenbeckia almawillia and $E$. cowanii belong to this subgenus.

Esenbeckia almawillia Kaastra, spec. nov., fig. 6.

Frutex 1-10 m, indumento e pilis fulvo-ferrugineis constanti. Folia alterna, subunifoliolata foliolo sessili. Petiolus supra planus, 4-10 mm longus, minute pubescens. Lamina folioli (anguste) elliptica vel (anguste) subobovata, basi anguste cuneata acuta vel obtusa vulgo aequali, apice obtuso-acuminato vel caudato-cuspidato acumine ipso emarginato $2-20 \mathrm{~mm}$ longo lateraliter curvato vel recto, 3-12 $\times 1.5-4.5 \mathrm{~cm}$, (sub)coriacea, utrinque griseo-viridis et subglabra. Inflorescentiae aliquot laterales vulgo foliis alternantes, unaquaeque $\mathrm{e}$ bracteae vel interdum folii axilla orta, e panicula dense contracta circa $5 \mathrm{~mm}$ diametro minute pubescenti floribus tribus ad octo subsessilibus constantes. Bracteae primariae caducae, anguste triangulares vel subulatae, 3-15 $\times$ circa $0.4-1.0 \mathrm{~mm}$, interdum lamina vestigiali. Pedunculus usque ad $2.5 \mathrm{~mm}$ longus, autem $4-13 \mathrm{~mm}$ in statu frugifero. Pedicelli usque ad $1.5 \mathrm{~mm}$ in statu frugifero crescentes. Bracteae ordine minore caducae, usque ad $5 \mathrm{~mm}$ longae. Flores 5-6 mm diametro. Calyx lobatus, circa 1.2-1.7 mm longus, lobis apertis (late) triangularibus subacutis $0.7-1.0 \times 0.5-1.0 \mathrm{~mm}$ infra minute strigosis. Petala valvata in aestivatione, ovata vel elliptica, acuta, $2.0-2.5 \times 1.0-1.2 \mathrm{~mm}$, coriacea, flavida, supra glabra vel papillosa, infra minute strigosa, unicostata. Discus cupulatus quinquelobatus tuberculo brevi in quoque lobo, glaber. Carpella connata, apophyse parva glandibus parvis obsita instructa, dense minute strigosa. Fructus depressi stellatim quinquelobati, $1.0-1.1 \mathrm{~cm}$ alti. Loculi apophyse dorso-apicali obtusa $2-4 \mathrm{~mm}$ longa instructi, pubescentes extus prominenter parallellinervii rugulosi minute tuberculati. Semen unum in quoque loculo, ovoideum, area chalazina manifesta. Embryo unus, punctatus.

Typus: E. Ule 9493, Bolivia, Pando, Río Acre, near Cobija, fl. \& fr., Dec. 1911 (HOLOTYPUS, K).

This new species differs strongly from all others in Esenbeckia, except for $E$. cowanii. The main differences are the lateral, reduced inflorescences which alternate with the foliage leaves, the valvate aestivation and the unicostate petals. The structure of the flowers, the fruits, and the seeds with embryo indicate a position within the Pilocarpinae, where the best place seems to be in the relatively polymorphic genus Esenbeckia.

It is a pleasure for me to dedicate this species to Dr. Alberta Maria Wilhelmina Mennega. She kindly assisted in solving some anatomical problems about this species, viz. when I initially had only one single specimen at my disposal. From 1946 until her retirement in August this year she has built up the wood collection of our institute, which is one of the most important ones in the world.

Esenbeckia cowanii Kaastra, spec. nov., fig. 7.

Arbor parva. Folia alterna, unifoliolata foliolo sessili. Petiolus semiteres, $7-10 \mathrm{~mm}$ longus, alis circa $0.2 \mathrm{~mm}$ latis in auriculam $2-4 \mathrm{~mm}$ longam terminan- 


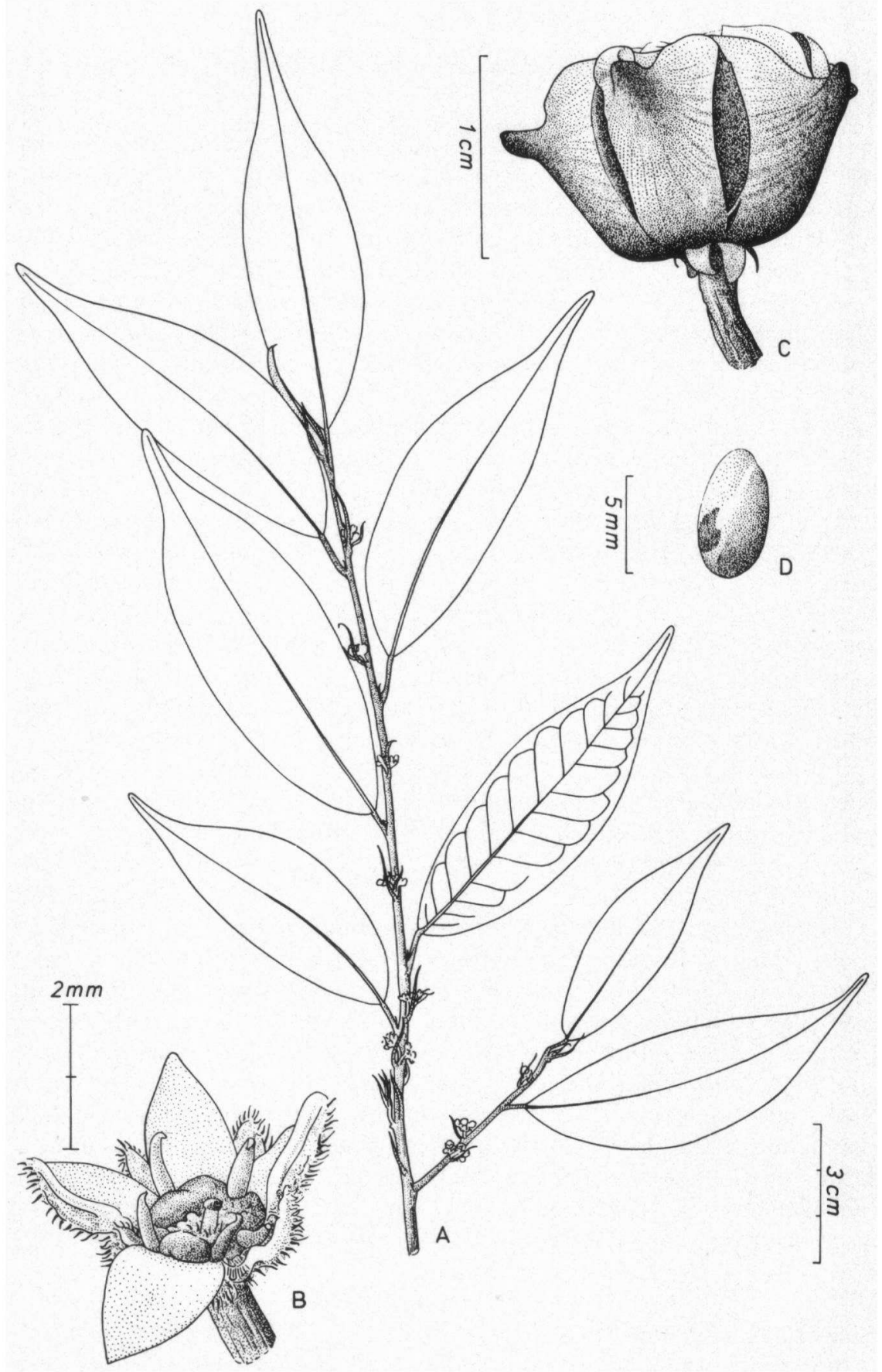

Fig. 6. Esenbeckia almawillia Kaastra. A. Flowering branchlet; B. flower, one petal removed, without anthers; C. fruit ; D. seed. A and B after Ule 9494 (G); C and D after d'Orbigny 764 (P). 


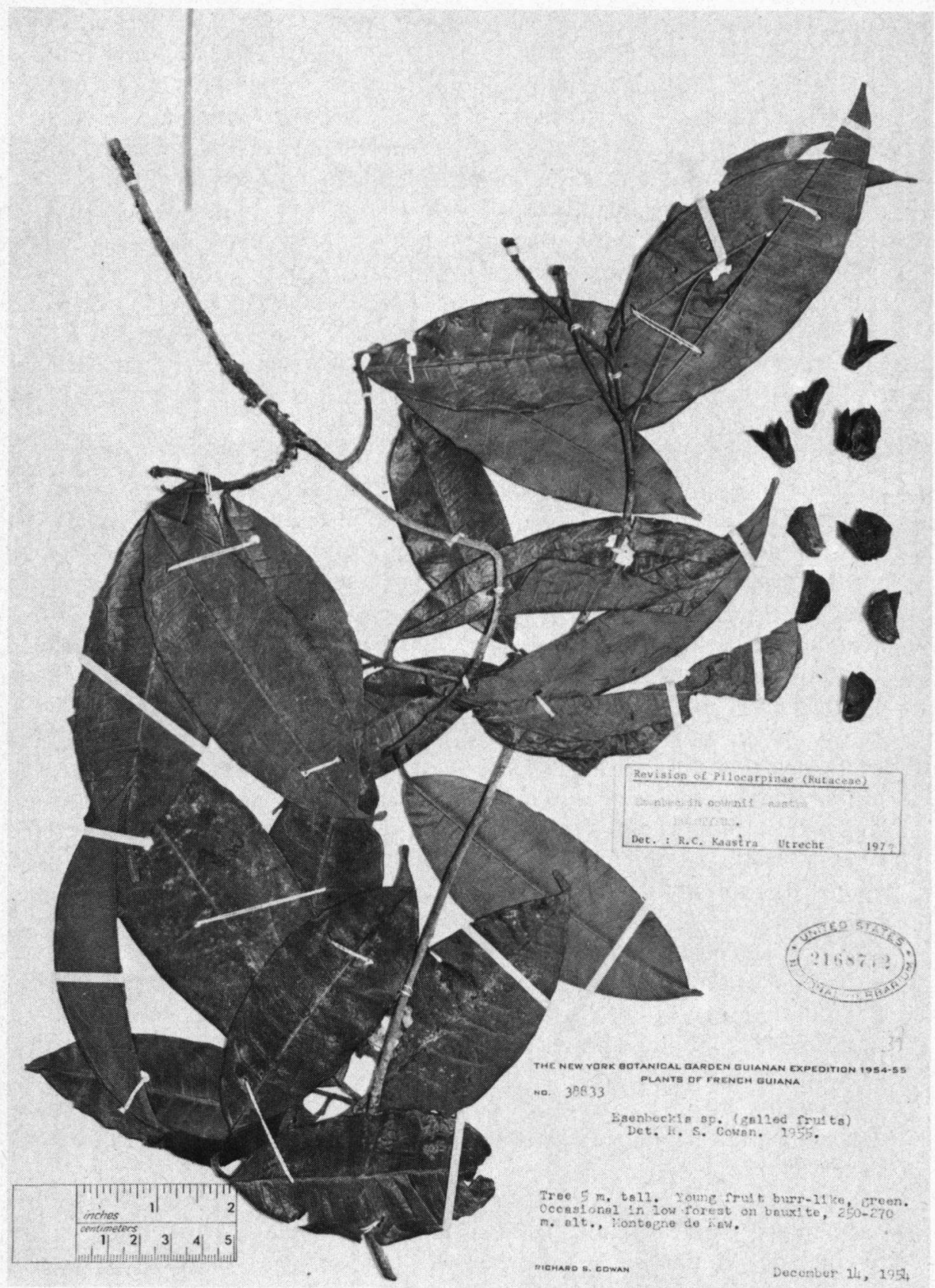

Fig. 7. Esenbeckia cowanii Kaastra, holotype (US). 
tibus. Lamina folioli (anguste) elliptica basi obtusa, apice acuminato acumine emarginato 5-7 mm longo, 6.5-18.0 × 2.5-7.0 cm, chartacea-subcoriacea, glabra praeter venam mediam basimque, vena media supra plana costataque. Infructescentiae laterales axillares plures paniculatae, foliis breviores. Calyx lobis depresse triangularibus coriaceis infra minute adpresse pubescentibus. Petala late ovata apice acuto, coriacea, indumento illius calycis simili. Fructus apicem versus leviter dilatati, $1.8-2.3 \mathrm{~cm}$ alti, paulo incani. Loculi apophyse dorsali obtusa 1-2 mm longa ad 1/3 usque ad 1/2 ab apice instructi. Semen oblique lacrimiforme, area chalazina manifesta nigra.

Typus: R. S. Cowan 38833, French Guiana, Guyane, Montagne de Kaw, alt. 250-270 m, fr., 14 Dec. 1954 (HoLotyPUS, US 2168712; isotypi, F, NY).

I dedicate this species to Dr. Cowan, the collector of the specimens. Like me, Dr. Cowan started his main botanical investigations in the Rutaceae. He has published several articles on this subject.

Unfortunately the flowers are badly known, but the position of this new species is most likely to be in subg. Lateriflorens. The fruits resemble those of $E$. leiocarpa, but they are more yellowish-hairy instead of greyish. Vegetatively both species cannot be mistaken. Many young fruits of the collections are transformed into galls with an echinoid appearance.

Metrodorea maracasana Kaastra, spec. nov., fig. 8.

Arbor parva, ramulis tenuibus glabris. Folia unifoliolata foliolo sessili vel obsolete petiolato. Petiolus semiteres late nec profunde canaliculatus, $1-2 \mathrm{~mm}$ longus, glaber, dorsifixus in vagina 4-7 mm longa. Lamina elliptica basi breviter angustata vel anguste subcuneata, apice obtuso, coriacea, glabra, vena media superne impressa. Inflorescentiae terminales late paniculatae floribus confertibus, pubescentes, pari ramulorum secundorum infimo pari foliorum supremo subtento, bracteis infimis quodam modo foliaceis. Flores circa $6 \mathrm{~mm}$ diametro. Lobi calycis aestivatione (sub)quincunciali, depresse ovati, infra minute pubescentes, pseudocosta indistincta instructi. Petala subquincuncialisubvalvata in aestivatione, elliptica apice acutiusculo, flavido-alba, utrinque minute pubescentia. Discus 5-10-lobatus, ovarium superans, subrasilis, glaber. Carpella immersa, glabra, tuberculis digitiformibus instructa, unumquidque post anthesin apophysem apicalem prodens. Stylus brevis stigmate capitato. Fructus ignoti.

Typus: E. Pereira 9656 \& G. F. J. Pabst 8545, Brazil, Bahia, between Itiruçu and Maracás, fl., 23 Jan. 1965 (HoLotyPus, M; isotypi, F 1629627, LP; omnes ex HB 35012).

Superficially $M$. maracasana resembles $M$. stipularis, but the petioles are partially free and the leaflets are exclusively unifoliolate. There is also some resemblance with $M$. nigra, but that species has usually longer pedicels, thinner leaves, and more slender flowering branchlets. $M$. maracasana is distinguished by its short petioles and the deeply immersed ovary which develops well-sized apophyses. 


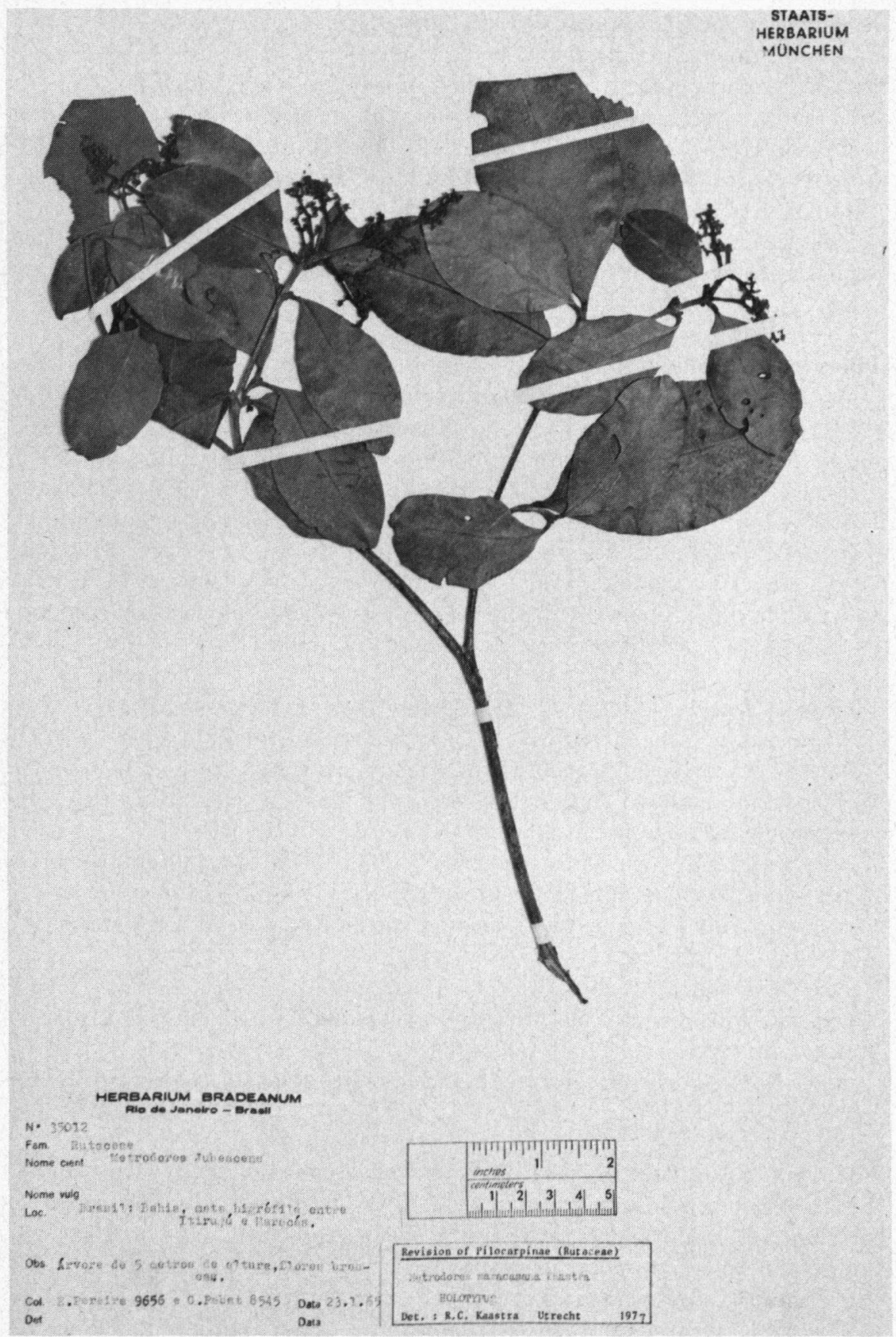

Fig. 8. Metrodorea maracasana Kaastra, holotype (M). 
Pilocarpus pennatifolius Lemaire var. pilosus Kaastra, var. nov.

Ramuli novelli petioli et raches pubescentes pilis patentibus $0.2-0.4 \mathrm{~mm}$ longis. Perulae gemmarum terminalium pilis usque $1 \mathrm{~mm}$ longis vestitae. Foliola (sub)coriacea, infra dense pubescentia pilis patentibus $0.2-0.5 \mathrm{~mm}$ longis.

Typus: Hj. Mosén 3954, Brazil, São Paulo, Campinas, fl. \& fr., Jul. 1875 (holotypus, S (fl.); isotypi, C $2 \times, \mathrm{LD}, \mathrm{P}$ (omnes ex S), S $4 \times$ (fl., fr., \& st., aliqui 23 vel 25 Iulio lecti).

This variety is distinctly hairy, especially the leaves below, while var. pennatifolius is nearly glabrous when adult. It has a separate area of distribution, mainly in São Paulo, where it is the only known variety.

Pilocarpus racemosus Vahl subsp. racemosus var. yucatanus Kaastra, var. nov.

Arbor parva. Perulae gemmarum terminalium dense pubescentes pilis 0.2 $0.3 \mathrm{~mm}$ longis. Folia (1-)3-foliolata vel imparipinnata 1-2-jugata. Petiolus, et basis venaque media foliolorum pubescens pilis $0.1-0.2 \mathrm{~mm}$ longis.

TyPus: G. F. Gaumer 24399, Mexico, Yucatán, st., 1917-1921 (HOLOTYPuS, F 552392; isotypi, G, GH, MO 951573, NY, US 1268394, omnes ex F).

Variety yucatanus is distinguished by its persistent indumentum. Var. racemosus is nearly always glabrous; an indumentum, if any, consists of hairs up to $0.05 \mathrm{~mm}$ only. There are no intermediate specimens known. Var. yucatanus is confined to Yucatán; var. racemosus is absent there.

Pilocarpus racemosus Vahl subsp. viridulus Kaastra, subsp. nov.

Arbor parva. Folia increbre (1-)2-3-jugata, vel 1-3-foliolata. Flores viriduli.

Typus: A. Jiménez M. 1529, Costa Rica, Guanacaste, Liberia, bank of the Río Colorado between Hacienda Guachipelín and Las Pailas, fl., 4 Jan. 1964 (HolotyPus, CR; isotypi, F 1698498, NY).

Subsp. viridulus has usually one pair of leaflets more than subsp. racemosus. Apart from this, it differs in the greenish flowers, which are purplish in var. racemosus. This subsp. is confined to Central America, and geographically isolated from the other subspecies.

Pilocarpus goudotianus Tulasne subsp. goudotianus var. mollis (Cuatrecasas) Kaastra, comb. nov.

Basionym: Pilocarpus alvaradoi Pittier var. mollis Cuatrecasas, Revista Acad. Colomb. Ci. Exact. 8: 467. 1952.

Pilocarpus goudotianus Tulasne subsp. heterochromus Kaastra, subsp. nov.

Arbor parva. Ramuli et perulae gemmarum terminalium glabrae. Petiolus subglaber. Racemi 3.0-3.5 cm diametro. Dentes calycis 0.3-0.5 × 1.0-1.1 mm, (sub)glabri. Petala infra versus basim violaceosuffusa et in parte apicali flavovirentia in sicco, cinnamomea apice ipso viridi in vivo, glabra.

Typus : S. Galen Smith 1136, Colombia, Huila, upper basin of Rio Magdalena, Cabrera Lajas, circa $11 \mathrm{~km}$ east of Villavieja on road to Baraya, fl. \& fr., 4 Jul. 1950 (HolotyPus, UC963230; isotypi, F 1435630, GH, MO, US 2047485). 
This taxon is distinguished by the glabrous branchlets and petioles, the smaller teeth of the calyx, and the colour of the petals. It is only known from the type collection.

Pilocarpus peruvianus (Macbride) Kaastra, stat. nov.

Basionym: Pilocarpus spicatus Saint-Hilaire var. peruvianus Macbride, Field Mus. Nat. Hist., Bot. Ser. 13: 676. 1949.

Pilocarpus spicatus Saint-Hilaire subsp. spicatus var. lealii (Machado) Kaastra, stat. nov.

Basionym: Pilocarpus lealii Machado, Bull. Mens. Soc. Linn. Lyon 16: 145, cum imagine luce impressa, 1947.

Typus: O. X. de Brito Machado RB 75557, Brazil, Rio de Janeiro, Restinga da Tijuca, fl. \& fr., 5 ·Feb. 1947 (LeCtotypus, RB (fl.); isolectotypi, RB 2×, US non vidi, WU (?) non vidi).

Pilocarpus spicatus Saint-Hilaire subsp. longeracemosus (Martius ex Engler in Martius) Kaastra, stat. nov.

Basionym: Pilocarpus longeracemosus Martius ex Engler in Martius, Fl. bras. 12(2): 135.1874.

Pilocarpus spicatus Saint-Hilaire subsp. aracatensis Kaastra, subsp. nov.

Arbor parva, puberula vel subglabra pilis usque ad $0.1 \mathrm{~mm}$ longis. Racemi usque ad $20 \times 1.0-1.5 \mathrm{~cm}$; pedicelli 4-5 mm longi. Flores pentameri, 4.5-5.2 $\mathrm{mm}$ diametro. Petala $2.0-2.3 \times 1.2-1.5 \mathrm{~mm}$. Filamenta subulata. Antherae versatiles, rectae.

Typus: T. N. Guedes 553, Brazil, Ceará, Serra da Aratanha, fl. \& fr., 27 Mar. 1958 (HOLOTYPUS, US 2484399; isotypi, NY, RB 114215 (sine numero, "26 Mar. 1958”, Ducke determinavit 1957!), omnes ex IAN).

This subspecies is closest to subsp. longeracemosus. It can be distinguished by the smaller flowers and the distinctly shorter hairs. It was discovered by Holmes, the curator of the Pharmaceutical Society of Great Britain, in 1895. He obtained some leaves and fruits of commerce and named them Aracati jaborandi, after the harbor in Cearà from which it was shipped to Liverpool. The insufficient material did not enable him to give it formal recognition. Now few flowering and fruiting collections are available, from the Serra da Aratanha in Cearà, Brazil.

Galipea lucida (Rusby) Kaastra, comb. nov.

Basionym: Esenbeckia lucida Rusby, Bull. New York Bot. Gard. 8: 98. 1912.

Typus: R. S. Williams 252, Bolivia, Río San Juan, fl./fr., 5 Apr. 1902 (ноLOTYPUS, NY; isotypi, BM, K, US).

Helietta mollis (Miquel) Kaastra, comb. nov.

Basionym: Esenbeckia mollis Miquel, Linnaea 22: 796.1850 (?) (“1849”, 
vide Stafleu [1966:90]); Engler in Martius, F1. bras. 12(2): 142. 1874.

TYPUS: J. S. Blanchet 3090, Brazil, Bahia, "marais de Tapira", fl., 1840 (holotypus, $\mathrm{U}$; isotypi, B (destructus; imago luce impressa, F, MO, NY). BM, BR $2 \times$, C, G, K $2 \times$, NY, P $3 \times$, W).

Synonym: Helietta puberula R. E. Fries, Bull. Herb. Boissier. ser. 2. 7: 1001. 1907. Syntypus vidi: J. D. Anisits 2049, Paraguay, Fuerte Olympo, fl., 12? Jan. 1898 (P ex S-herb. Regnell).

Some of the type specimens of Esenbeckia mollis show young fruits with the typical wings of Helietta already developed. The one type specimen of Helietta puberula which I studied appears to have pentamerous flowers as well as tetramerous ones. The calyx lobes are not glabrous, but show a distinct pubescence, although this is less dense than in E. mollis, generally. The differences between $H$. puberula and E. mollis, according to the descriptions, do not exist, therefore both taxa are conspecific.

\section{ACKNOWLEDGEMENTS}

I wish to thank Mr. L. Y. Th. Westra for his critical remarks on some of the Latin descriptions, Mr. A. Kuiper for the photographs, and Mr. H. R. Rijpkema for the drawing. Thanks are due to the Directors and Keepers of the herbaria, who enabled me to study the specimens. Herbarium studies in Genève, Kew, London (British Museum [Natural History]), and Paris were supported by a grant from the Netherlands Organization for the Advancement of Pure Research (Z.W.O.).

\section{REFERENCE}

Stafleu, F. A. (1966): F. A. W. Miquel, Netherlands botanist. Wentia 16: 1-95. 